\title{
Assessment of post-traumatic stress disorder in front-line and non-front-line medical staff with COVID-19 patients: a cross-sectional study in Iran
}

\author{
SAEEDEH ASKARI 1, A, B, D, MARYAM BEHESHTINASAB ${ }^{1, A, ~ B, ~ D, ~ S A E E D ~ G H A N B A R I ~ 2, ~ c, ~ D, ~}$ \\ HADIS BAHMAEI 1, A, B, D , HATAM BOOSTANI 3, A, F, POORANDOKHT AFSHARI 1, A, D, F, G,
}

PARVIN ABEDI $\left.\right|^{4, A, D, E}$

ORCID ID: 0000-0002-6980-0693

${ }^{1}$ Reproductive Health Promotion Research Center, Ahvaz Jundishapur University of Medical Sciences, Ahvaz, Iran

${ }^{2}$ Biostatistics Department, Ahvaz Jundishapur University of Medical Sciences, Ahvaz, Iran

${ }^{3}$ Psychiatry Department, Ahvaz Jundishapur University of Medical Sciences, Ahvaz, Iran

${ }^{4}$ Midwifery Department, Menopause Andropause Research Center, Ahvaz Jundishapur University of Medical Sciences, Ahvaz, Iran

A - Study Design, B - Data Collection, C - Statistical Analysis, D - Data Interpretation, E - Manuscript Preparation, F - Literature Search, G - Funds Collection

Summary Background. Post-traumatic stress disorder (PTSD) is a disorder that can occur after exposure to a traumatic event. Objectives. This study is designed to evaluate PTSD in front-line and non-front-line medical staff who provide care for COVID-19 patients.

Material and methods. This cross-sectional study was conducted on 200 medical staff in two hospitals of Ahvaz. The inclusion criterion was having more than six months of service. Health providers with psychological disorders, a history of crises in the past six months or divorced subjects were excluded from the study. A demographic questionnaire and the PTSD questionnaire were completed by participants. The Independent $t$-Test, chi-square test and linear regression were used to analyze the data.

Results. The total score of PTSD was $50.08 \pm 12.24$ and $44.06 \pm 11.32$ in front-line and non-front-line medical staff, respectively $(p<$ 0.001 ). $10 \%$ and $1 \%$ of front-line and non-front-line medical staff had severe PTSD, respectively. Females were 7.47 times more likely to have PTSD compared to males. Medical staff who had a child were 6 times more likely to have PTSD compared with those without a child. Medical staff involved in the care of COVID-19 patients were 5.67 times more likely to have PTSD $(p<0.001)$. Health providers whose relatives died from COVID-19 were 10.59 times more likely to have PTSD.

Conclusions. The results of this study showed that front-line medical staff are more susceptible to PTSD in comparison to non-frontline medical staff. Health policymakers should pay attention to the psychological health of medical staff in times of crises such as the COVID-19 pandemic.

Key words: COVID-19, medical staff, post-traumatic stress disorder.

Askari S, Beheshtinasab M, Ghanbari S, Bahmaei H, Boostani H, Afshari P, Abedi P. Assessment of post-traumatic stress disorder in front-line and non-front-line medical staff with COVID-19 patients: a cross-sectional study in Iran. Fam Med Prim Care Rev 2021; 23(2): 139-143, doi: https://doi.org/10.5114/fmpcr.2021.105904.

\section{Background}

The first case of COVID-19 was recognized in Iran in March 2020. The number of COVID-19 cases is 703,288 , and 39,202 cases have died from this disease in Iran [1]. The COVID-19 pandemic has led to a significantly large number of psychological consequences. Negative feelings (such as anxiety, depression and anger) increase sensitivity to social risks, while positive emotions and life satisfaction have decreased [2].

Post-traumatic stress disorder (PTSD) is characterized by re-experiencing a traumatic event, avoiding accident-reminiscent stimuli and excessive arousal and negative changes in cognition and mood. The Fifth Diagnostic and Statistical Manual of Mental Disorders identifies PTSD as part of a set of diagnostic, traumatic and stress-related disorders that can occur after exposure to a traumatic event [3]. PTSD has many overlaps with other psychiatric disorders that cause a person to endure ad- ditional stress in addition to the injury [4]. Its main characteristics are severe stress and anxiety after a traumatic or stressful event, which causes the person with this experience to react with fear and helplessness and try not to remember the whole thing or forget parts of it. People with this disorder re-experience the traumatic event in their dreams, as well as in their daily thoughts. These people show symptoms including depression, anxiety and cognitive problems, such as poor concentration [5]. Patients with diseases such as acquired immunodeficiency syndrome (AIDS) [6], hepatitis [7], heart disease [8] and burns [9] have also experienced PTSD. In Spain, the results of a study showed that the prevalence of depression, anxiety and PTSD is $18.7 \%, 21.6 \%$ and $15.8 \%$, respectively. In elderly people, having economic stability and the belief that sufficient information about the epidemic was provided by health care providers was negatively associated with depression, anxiety and PTSD [10]. PTSD may affect patients with COVID-19 and the health professionals responsible for the care of COVID-19 patients [11]. 
Huang et al., in their study which aimed to assess the mental health of front-line personnel in the fight against coronavirus, showed that the prevalence of anxiety and stress disorders in women was higher than in men [11]. Shechtera et al. found that health professionals who cared for COVID-19 patients experienced $57 \%$ PTSD, $48 \%$ depression and $33 \%$ anxiety [12].

COVID-19 is an unknown infectious disease, the angles of which are not yet known. It is not clear when humans will be able to control this disease.

\section{Objectives}

This study aimed to explore the relationship between COVID-19 and PTSD for front-line and non-front-line medical staff who provided care for COVID-19 patients.

\section{Material and methods}

\section{Study design and participants}

This was a cross-sectional study that was conducted in the Razi and Sina hospitals, where the first hospital is the main center for admission of COVID-19 patients, and the second is for non-COVID-19 patients in Ahvaz, Iran.

Using Med Calc software with a $90 \%$ and $95 \%$ confidence interval, a total number of $\mathbf{2 0 0}$ medical staff were considered for the sample size (100 in each group of front-line and non-front-line medical staff).

The inclusion criterion was having more than six months service. Medical staff with psychological disorders such as anxiety, stress or depression before starting work in their current position, with a history of crises in the past six months, death of close relatives or being divorced were excluded from the study.

\section{Measures}

A demographic questionnaire and the PTSD questionnaire were completed by the participants.

The demographic questionnaire contained questions about age, gender, education, health profession studies, whether or not they have a child and whether or not somebody has died in their family because of COVID-19.

The Post-traumatic Stress Disorder Checklist (PCL-5) was used to collect data about PTSD. This questionnaire contains four sub- scales that precisely match four signs of DSM-5, including re-experiencing (items $1-5$ ), avoidance (items $6-7$ ), negative alteration in cognition and mood (items 8-14) and hyperarousal (items 15-20). This questionnaire can be used for the diagnosis of PTSD, as well as to monitor symptom changes. A five-item Likert scale was used for scoring, with 0 used for "not at all", and 4 used for "severe symptoms". The total score of this scale varied from 0 to 80 , and the cut-off score was 38 for a provisional diagnosis of PTSD. The scores of the PLC- 5 checklist was classified as follow: score < 33 was negative for PTSD, a score between $34-48$ was mild PTSD, a score between 49-63 was moderate PTSD, and a score between 64-80 was severe PTSD [13]. The validity and reliability of this scale have been approved in Iran [14]. All respondents requested to complete the demographic and the PCL-5 questionnaires.

\section{Ethical approval}

The protocol of this study was approved in the Ethics Committee of Ahvaz Jundishapur University of Medical Sciences (IR.AJUMS.REC.1399.080). All participants provided written informed consent prior to data collection.

\section{Statistics}

All data was entered in STATA version 11. The normal distribution of data was assessed using the Shapiro-Wilk test. The Independent $t$-Test and chi-square test was used for checking the differences in the two groups regarding continuous and categorical data. Linear regression was used to determine the effect of independent variables on PTSD. $P<0.05$ was considered significant.

\section{Results}

In this study, 200 medical staff were recruited, in which 100 were involved in the care of COVID-19 patients, and 100 medical staff did not have contact with COVID-19 patients. Table 1 shows the demographic characteristics of the participants. Most of the participants in the two groups were female, with a mean age of $35.23 \pm 8.1$ and $34.54 \pm 7.8$ years in the two groups of front-line and non-front-line staff for COVID-19 patients, respectively. The two groups did not have any significant difference regarding education, having a child and the field of education (Table 1 ).

\begin{tabular}{|c|c|c|c|}
\hline Variable & $\begin{array}{l}\text { Involved in the care of COVID-19 patients } \\
n=100\end{array}$ & $\begin{array}{l}\text { Not involved in the care of COVID-19 patients } \\
n=100\end{array}$ & $p$ \\
\hline & \multicolumn{2}{|l|}{ Mean \pm SD or $n(\%)$} & \\
\hline Age $(\mathrm{y})$ & $35.23 \pm 8.1$ & $34.54 \pm 7.8$ & 0.54 \\
\hline Having a child & $64(64)$ & $54(54)$ & 0.15 \\
\hline Death of relatives due to COVID-19 & $17(17)$ & $19(19)$ & 0.71 \\
\hline $\begin{array}{c}\text { Gender } \\
\text { male } \\
\text { female } \\
\end{array}$ & $\begin{array}{l}38(38) \\
62(62)\end{array}$ & $\begin{array}{l}39(39) \\
61(61)\end{array}$ & 0.88 \\
\hline $\begin{array}{l}\text { Field of health care } \\
\text { nursing } \\
\text { MD } \\
\text { midwifery } \\
\text { laboratory technician } \\
\text { operating room technician } \\
\text { emergency medical technician } \\
\text { radiologist } \\
\text { anesthesiologist } \\
\text { secretory } \\
\text { others }\end{array}$ & $\begin{array}{l}8(8) \\
15(15) \\
7(7) \\
8(8) \\
10(10) \\
6(6) \\
8(8) \\
8(8) \\
7(7) \\
14(14)\end{array}$ & $\begin{array}{l}10(10) \\
12(12) \\
6(6) \\
11(11) \\
10(10) \\
5(5) \\
10(10) \\
6(6) \\
9(9) \\
13(13)\end{array}$ & 0.99 \\
\hline
\end{tabular}




\begin{tabular}{l}
$\begin{array}{l}\text { Table 1. Demographic characteristics of participants in two groups of front-line and non-front-line medical staff during the COVID-19 } \\
\text { pandemic }\end{array}$ \\
\begin{tabular}{|l|l|l|l|}
\hline Variable & $\begin{array}{l}\text { Involved in the care of COVID-19 patients } \\
n=100\end{array}$ & $\begin{array}{l}\text { Not involved in the care of COVID-19 patients } \\
n=100\end{array}$ & $p$ \\
\hline & Mean \pm SD or $n(\%)$ & & \\
\hline $\begin{array}{l}\text { Education } \\
\text { diploma }\end{array}$ & $9(9)$ & $\begin{array}{l} \\
\text { associate degree }\end{array}$ & $9(12)$ \\
bachelor & $9(9)$ & $61(61)$ \\
master & $60(60)$ & $6(6)$ \\
PhD or MD & $7(7)$ & $12(12)$ \\
\hline
\end{tabular} \\
\hline
\end{tabular}

\begin{tabular}{|l|l|l|l|}
\hline \multicolumn{3}{|l|}{ Table 2. Scores of PTSD in the two groups of front-line and non-front-line medical staff during the COVID-19 pandemic } \\
\hline Variables & $\begin{array}{l}\text { Involved in the care of COVID-19 } \\
\text { patients } \\
n=100\end{array}$ & $\begin{array}{l}\text { Not involved in the care of COVID-19 } \\
\text { patients } \\
n=100\end{array}$ & $p$ \\
\hline Re-experiencing & $7.82 \pm 3.8$ & $6.39 \pm 3.3$ & 0.005 \\
\hline Avoidance & $4.56 \pm 1.95$ & $4.1 \pm 1.86$ & 0.09 \\
\hline Negative alteration in cognition and mood & $11.29 \pm 2.6$ & $10.25 \pm 2.2$ & 0.003 \\
\hline Hyperarousal & $10.56 \pm 3.45$ & $9.14 \pm 3.3$ & 0.003 \\
\hline PTSD total score & $50.08 \pm 12.24$ & $44.06 \pm 11.32$ & $<0.001$ \\
\hline
\end{tabular}

\begin{tabular}{|l|l|l|l|}
\hline \multicolumn{5}{|l|}{ Table 3. Frequency of PTSD in the two groups with and without direct contact with COVID-19 patients } \\
\hline Variables & $\begin{array}{l}\text { Involved in the care of COVID-19 patients } \\
n=100\end{array}$ & $\begin{array}{l}\text { Not involved in the care of COVID-19 patients } \\
n=100\end{array}$ & $p$ \\
\hline & $n(\%)$ & $82(82)$ & 0.06 \\
\hline Medical staff with PTSD & $91(91)$ & $18(18)$ & 0.001 \\
\hline Negative for PTSD & $9(9)$ & $42(42)$ & $39(39)$ \\
\hline Mild PTSD & $31(31)$ & $1(1)$ & \\
\hline Moderate PTSD & $50(50)$ & & \\
\hline Severe PTSD & $10(10)$ & & \\
\hline
\end{tabular}

\begin{tabular}{|l|l|l|l|l|}
\hline \multicolumn{5}{|l|}{ Table 4. Linear and multiple logistic regression for detecting the relationship between PTSD and other variables } \\
\hline PTSD & Coefficients & St-Error & 6 & $p$ \\
\hline Gender (female) & 7.47 & 1.48 & 0.3001793 & $<001$ \\
\hline Having child & -6.15 & 1.93 & -0.2497728 & 0.002 \\
\hline Group & -5.67 & 1.39 & -0.2343041 & $<001$ \\
\hline Death of relatives & -10.59 & 1.86 & -0.3361345 & $<001$ \\
\hline Age & -0.219 & 0.121 & -0.1440876 & 0.072 \\
\hline
\end{tabular}

Female compared to male.

Having child compared to not having a child.

Direct contact compared to indirect contact with COVID-19 patients.

Death of relatives due to COVID-19 compared to those whose relatives did not.

Table 2 shows the mean score of PTSD in the two groups with and without direct contact with COVID-19 patients. As evident from this table, the mean score of re-experiencing, avoidance, negative alteration in cognition and mood and hyperarousal was significantly higher in the medical staff with direct contact with COVID-19 patients $(p<0.05)$. The total score of PTSD was 50.08 \pm 12.24 and $44.06 \pm 11.32$ in front-line and non-front-line medical staff, respectively $(p<0.001)$.

Table 3 shows the frequency of PTSD in medical staff. As can be seen, $91 \%$ of front-line medical staff had PTSD, while $82 \%$ of non-front-line staff had PTSD ( $p=0.06) .10 \%$ and $1 \%$ of health providers with and without direct contact with COVID-19 had severe PTSD, respectively ( $p=0.001)$.

Table 4 evaluates the effect of independent variables on the PTSD score using linear regression. Females were 7.47 times more likely to have PTSD compared to males $(p<0.001)$. People who had a child were 6 times more likely to have PTSD compared with those without a child $(p=0.002)$. Health providers involved in the care of COVID-19 patients were 5.67 times more likely to have PTSD $(p<0.001)$. Health providers whose relatives died from COVID-19 were 10.59 times more likely to have PTSD compared to those whose relatives did not $(p<0.001)$.

\section{Discussion}

This study was designed to evaluate the relationship between COVID-19 and PTSD in front-line and non-front-line medical staff. Our results showed that the mean score of all sub-scales of PTSD, including re-experiencing, avoidance, negative alteration in cognition and mood and hyperarousal, was significantly higher in front-line medical staff. The total score of PTSD was also significantly higher in front-line medical staff. Other studies have also confirmed our results. Lai et al., in a study, found that $71.5 \%$ of participants who provided care of COVID-19 patients reported mild to severe PTSD [15].

$\mathrm{Li}$ et al., in a study on 214 individuals from the general population and 234 front-line nurses with COVID-19 patients and 292 non-front-line nurses, found that the physiological and psycho- 
logical scores for front-line nurses were significantly lower than that of non-frontline nurses and the general population [16].

Our results showed that $91 \%$ of front-line medical staff had PTSD, while $82 \%$ of staff without direct contact had PTSD. More front-line medical staff also had severe PTSD. Qianian et al., in a study on $\mathbf{3 7 7}$ front-line health providers, found that the prevalence of post-traumatic stress symptoms (PTSS) was 3.8\%, and females were more likely to have PTSS, with a hazard ratio of 2.13 [17]. Huang et al., in their study on 246 medical staff who provided care of COVID-19 patients, found that $23 \%$ of the staff suffered from anxiety, and the score of anxiety and PTSD-SS in females was significantly higher than that in male staff [18]. Our results showed a higher rate of PTSD in medical staff. This discrepancy may result from the fact that the Iranian medical staff did not access to protective equipment because of sanctions, and this matter may cause more stress and anxiety for them.

Our findings showed that females were 7.47 times more likely to have PTSD compared to males. Ainamani et al. [19], in their study, also found that among 325 male and female refugees that experienced war, $94 \%$ of women and $84 \%$ of men suffered from PTSD.

People who had a child were 6 times more likely to have PTSD compared with those without a child. Health providers involved in the care of COVID-19 patients were 5.67 times more likely to have PTSD. Health providers whose relatives died from COVID-19 were 10.59 times more likely to have PTSD compared to those whose relatives did not. Johnson et al., in their study, found that $28.9 \%$ of health providers had PTSD, and those who worked directly with COVID-19 patients had higher PTSD and depression [20]. Si et al., in their study on 863 medi- cal care workers in China, found that $40.2 \%$ of the participants had post-traumatic stress, where $8.6 \%$ of them had severe PTS. They concluded that social support and active coping strategies could decrease the score of PTS [21]. The results of Johnson and Si's studies are different from our results. The reason for this discrepancy is that health providers in Iranian hospitals had a shortage of protective equipment, and they also did not receive sufficient social support. These factors may increase their stress, as well as PTSD.

\section{Strengths and limitations of the study}

This is the first time that we have evaluated PTSD in frontline and non-front-line medical staff of COVID-19 patients in Iran. Iran is a country that has encountered many natural disasters, such as floods and earthquakes, in recent years. Policymakers can use the results of this study to make a plan for considering the psychological care of health care providers.

Despite these strengths, this study has some limitations. We recruited health providers with different majors of studies. If we considered one field of health providers, such as nurses, the generalizability of findings could be better.

\section{Conclusions}

The results of this study showed that front-line medical staff are more susceptible to PTSD in comparison to non-front-line medical staff. Health policymakers should pay attention to the psychological health of medical staff in times of crises such as the COVID-19 pandemic.

Source of funding: This study was funded by Ahvaz Jundishapur University of Medical Sciences, Ahvaz, Iran.

Conflicts of interest: The authors declare no conflicts of interest.

\section{References}

1. Worldometers. Coronavirus [cited 10.11.2020]. Available from URL: https://www.worldometers.info/coronavirus/country/iran/.

2. Li S, Wang Y, Xue J, et al. The impact of COVID-19 epidemic declaration on psychological consequences: a study on active Weibo users. Int J Environ Res Public Health 2020; 17: 2032, doi: 10.3390/ijerph17062032.

3. Resick PA, Bovin MJ, Calloway AL, et al. A critical evaluation of the complex PTSD literature: implications for DSM-5. J Traum Stress 2012; 25: 241-251.

4. Pietrzak RH, Goldstein RB, Southwick SM, et al. Prevalence and axis I comorbidity of full and partial posttraumatic stress disorder in the United States: results from Wave 2 of the National Epidemiologic Survey on Alcohol and Related Conditions. J Anxiety Disord 2011; 25: 456-465.

5. Esmkhani H, Etemadi A, Nasirnejad A. Effectiveness of group narrative therapy on anxiety and depression of PTSD patients. J New Adv Behav Sci 2015; 7: 1-10.

6. Machtinger EL, Wilson T, Haberer JE, et al. Psychological trauma and PTSD in HIV-positive women: a meta-analysis. AIDS Behav 2012; 16: 2091-2100.

7. Burroughs TK, Wade JB, Ellwood MS, et al. Effect of post-traumatic stress disorder on cognitive function and covert hepatic encephalopathy diagnosis in cirrhotic veterans. Digest Dis Sci 2018; 63: 481-485.

8. Mirzaee MS, Hajivandi A, Khalili A, et al. The effect of poetry therapy on post-traumatic stress disorder (PTSD) in myocardial infarction patients. IJPN 2016; 4: 11-16.

9. Kafi S, Atashkar S, Amir AS, et al. Relationship of post-traumatic stress disorder with psychological defence styles in burn patients. Horizon Med Sci 2013; 19: 155-160.

10. González-Sanguinoa C, Ausína B, Ángel Castellanosb M, et al. Mental health consequences during the initial stage of the 2020 Coronavirus pandemic (COVID-19) in Spain. Brain Behav Immun 2020; 87: 172-176.

11. Huang J, Han M, Luo T, et al. [Mental health survey of 230 medical staff in a tertiary infectious disease hospital for COVID-19]. Zhonghua Lao Dong Wei Sheng Zhi Ye Bing Za Zhi 2020; 38: 192-195 (in Chinese).

12. Shechtera A, Diaza F, Moisea ND, et al. Psychological distress, coping behaviors, and preferences for support among New York healthcare workers during the COVID-19 pandemic. Gen Hosp Psychiatry 2020; 66: 1-8.

13. Weathers FW, Litz BT, Keane TM, et al. The PTSD checklist for DSM-5 (PCL-5). Available from the URL: www.ptsd.va.gov.

14. Varmaghani H, Fathi-Ashtiani A, Poursharifi H. Psychometric properties of the Persian Version of the posttraumatic stress disorder checklist for DSM-5 (PCL-5). Appl Psychol Res 2018; 9(3): 131-142.

15. Lai J, Ma S, Wang $\mathrm{Y}$, et al. Factors associated with mental health outcomes among health care workers exposed to coronavirus disease 2019. JAMA 2020; 3: e203976, doi: 10.1001/jamanetworkopen.

16. Li Z, Ge J, Yang M, et al. Vicarious traumatization in the general public, members, and non-members of medical teams aiding in COVID-19 control. Brain Behav Immun 2020; 88: 916-919, doi: 10.1016/j.bbi.2020.03.007. 30309-3.

17. Qianian Y, Sun Z, Liu T, et al. Posttraumatic stress symptoms of health care workers during the Corona Virus Disease 2019 (COVID-19). Clin Psychol Psychother 2020; 27: 384-395, doi: 10.1002/cpp.2477. 
18. Huang JZ, Han TD, Ren AK, et al. [Mental health survey of medical staff in a tertiary infectious disease hospital for COVID-19]. Zhonghua Lao Dong Wei Sheng Zhi Ye Bing Za Zhi 2020; 38: 192-195 (in Chinese).

19. Ainamani HE, Elbert T, Olema DK, et al. Gender differences in response to war-related trauma and posttraumatic stress disorder-a study among the Congolese refugees in Uganda. BMC Psychiatry 2020; 20: 17, doi: 10.1186/s12888-019-2420-0.

20. Johnson SU, Ebrahimi O, Hoffart A. PTSD symptoms among health workers and public service providers during the COVID-19 outbreak. PIoS ONE 2020; 15(10): e0241032, doi: 10.1371/journal.pone.0241032.

21. Si MY, Su XY, Jiang Y, et al. Psychological impact of COVID-19 on medical care workers in China. Infec Dis Poverty 2020; 113, doi: 10.1186/s40249-020-00724-0.

Tables: 4

Figures: 0

References: 21

Received: 20.11.2020

Reviewed: 15.12 .2020

Accepted: 17.03.2021

Address for correspondence:

Poorandokht Afshari, PhD

Reproductive Health Promotion Research Center

Ahvaz Jundishapur University of Medical Sciences

Ahvaz

Iran

Tel.: +989166464984

E-mail: poafshari@gmail.com 